\title{
Die Beeinflussung des Ionisationsgrades durch Diffusions- und Massenströme im Plasma *
}

\author{
K. BERgstedt \\ Institut für Plasmaphysik, 8046 Garching b. München, Germany \\ (Z. Naturforsch. 24 a, 299-310 [1969] ; eingegangen am 30. November 1968)
}

\begin{abstract}
A stationary, magnetically stabilized helium arc plasma is taken as an example to show that ionization degrees in such plasmas with large electron temperature and electron density gradients may not be calculated from static ionization relations valid for homogeneous plasmas (e. g. the Saha equation). Instead, ionization degrees in inhomogeneous plasmas must be determined from continuity equations of the form $\operatorname{div}\left(n_{Z} \boldsymbol{v}_{Z}\right)=J_{Z-1} n_{Z-1}-R_{Z} n_{Z}$ where $J_{Z-1}$ and $n_{Z-1}$ are the ionization rate and the density of the $Z-1$ times charged ions, and $R_{Z}, n_{Z}$ and $\boldsymbol{v}_{Z}$ are the recombination rate, the density and the centre-of-mass velocity of the $Z$ times charged ions. Static ionization formulae may not be applied to inhomogeneous plasmas because the finite relaxation times for the attainment of static ionization equilibria result in these ionization equilibria being displaced by the motion of the ions parallel to the direction of electron temperature and density gradients.

This is proved by means of two independent operations: firstly, the velocities of the helium ions, these being governed primarily by ambipolar diffusion, are calculated with the spectroscopically measured state variables from the momentum equations of the plasma; secondly, the velocities of the doubly charged ions are determined by integrating the continuity equation for these ions, use again being made of the measured state variables and of the ionization and recombination rates $J_{1}$ and $R_{2}$ of the helium ions that were calculated for this special plasma. The agreement between the independently obtained velocity values proves that the degree of ionization of the helium ions in this inhomogeneous plasma is described not by a static ionization formula, but by the continuity equations for these ions.

Furthermore, it is shown that the ionization "equilibria" between singly charged and neutral helium particles and between three times (CIV) and four times (CV) charged carbon particles are not determined by means of static ionization formulae either.

The influence of this hitherto disregarded effect on the spectroscopic determination of the electron temperature in the plasma discussed is illustrated in a diagram.
\end{abstract}

Bei der spektroskopischen Temperaturbestimmung aus dem Intensitätsverhältnis von Spektrallinien zweier Ionensorten desselben Elements nimmt man normalerweise an, daß das Verhältnis der Teilchendichten in den beiden (aufeinanderfolgenden) Ionisationsstufen dieses „Meßelements" auch in einem inhomogenen Plasma an jedem Ort durch eine statische Ionisationsformel bestimmt ist. Man nimmt also keine Rücksicht darauf, daß die in einem inhomogenen Plasma im allgemeinen vorhandenen Diffusionsströme von Ionen der einen oder anderen Art oder auch Plasmaströmungen einen Einfluß auf das Verhältnis der Ionendichten nehmen können; denn man vernachlässigt die endliche Einstellzeit des Gleichgewichts an jedem Ort. Dieses ist sicher in vielen praktischen Fällen gerechtfertigt. Wenn aber die Temperatur- und damit auch die Ionendichtegradienten hinreichend groß werden, hat man die bei der Einstellung des Gleichgewichts auftretenden Relaxa-

* Auszug aus der von der Fakultät für Maschinenwesen und Elektrotechnik der Technischen Hochschule München genehmigten Dissertation. tionserscheinungen unbedingt $\mathrm{zu}$ berücksichtigen. Das soll hier am Beispiel einer magnetisch stabilisierten Bogenentladung demonstriert werden.

Nimmt man an, daß im betrachteten Plasmabereich nur $(Z-1)$ - und $Z$-fach geladene Ionen eines Elements auftreten und bezeichnet man mit $J_{Z-1}$ die Ionisationsrate der $(Z-1)$-fach geladenen Ionen und mit $R_{Z}$ die Rekombinationsrate der $Z$-fach geladenen Ionen, so erhält man die Kontinuitätsgleichungen für beide Ionensorten unter Annahme der Stationarität in der Form:

$$
\begin{aligned}
& J_{Z-1} n_{Z-1}-R_{Z} n_{Z}=\operatorname{div}\left(n_{Z} \boldsymbol{v}_{Z}\right) \\
\text { und } \quad R_{Z} n_{Z}-J_{Z-1} n_{Z-1} & =\operatorname{div}\left(n_{Z-1} \boldsymbol{v}_{Z-1}\right) .
\end{aligned}
$$

Dabei sind $n_{Z}$ und $n_{Z-1}$ die Teilchendichten und $\boldsymbol{v}_{Z}$ und $\boldsymbol{v}_{Z-1}$ die Schwerpunktsgeschwindigkeiten beider Ionenkomponenten.

Für den „statischen“ Fall gilt:

$$
J_{Z-1} n_{Z-1}-R_{Z} n_{Z}=0 .
$$

Im lokalen thermodynamischen Gleichgewicht ergibt sich aus Gl. (2) die Saha-Gleichung. 
Das im folgenden als Ionisationsverhältnis bezeichnete Verhältnis $n_{Z} / n_{Z-1}$ der Ionendichten erhält man aus den Gln. (1 a) und ( 1 b) :

$$
\begin{aligned}
\frac{n_{Z}}{n_{Z-1}} & =\frac{J_{Z-1}}{R_{Z}}-\frac{\operatorname{div}\left(n_{Z} \boldsymbol{v}_{Z}\right)}{R_{Z} n_{Z-1}}=\frac{J_{Z-1}}{R_{Z}}+\frac{\operatorname{div}\left(n_{Z-1} \boldsymbol{v}_{Z-1}\right)}{R_{Z} n_{Z-1}} \\
& =\frac{J_{Z-1}}{R_{Z}}\left(1-\frac{\operatorname{div}\left(n_{Z} \boldsymbol{v}_{Z}\right)}{J_{Z-1} n_{Z-1}}\right) \\
& =\frac{J_{Z-1}}{R_{Z}}\left(1+\frac{\operatorname{div}\left(n_{Z-1} \boldsymbol{v}_{Z-1}\right)}{J_{Z-1} n_{Z-1}}\right)
\end{aligned}
$$

während sich für den statischen Fall aus Gl. (2)

$$
n_{Z} / n_{Z-1}=J_{Z-1} / R_{Z}
$$

ergibt. Demnach kann man den Einfluß der Ionenbewegung auf die Einstellung des örtlichen Ionisationsgleichgewichts im Plasma dann vernachlässigen, wenn

$$
\left|\operatorname{div}\left(n_{Z} \boldsymbol{v}_{Z}\right)\right| / J_{Z-1} n_{Z-1} \ll 1
$$

oder

$$
\begin{aligned}
& \text { wegen } \operatorname{div}\left(n_{Z} \boldsymbol{v}_{Z}\right)=-\operatorname{div}\left(n_{Z-1} \boldsymbol{v}_{Z-1}\right) \\
& \left|\operatorname{div}\left(n_{Z-1} \boldsymbol{v}_{Z-1}\right)\right| / J_{Z-1} n_{Z-1} \ll 1
\end{aligned}
$$

sind.

In dieser Arbeit werden die Relaxationseffekte bei der Ionisation in einer quasistationären Heliumentladung, wie sie von WuLFF ${ }^{1}$ entwickelt wurde, experimentell untersucht. Im ersten Abschnitt wird die Versuchsanordnung skizziert und im zweiten Abschnitt die Messung der Zustandsgrößen besprochen, die hier natürlich ohne die Benutzung einer statischen Ionisationsbeziehung ermittelt werden. Die Schwerpunktsgeschwindigkeiten $\boldsymbol{v}_{1}$ und $\boldsymbol{v}_{2}$ der einfach und zweifach geladenen Ionen werden im dritten Abschnitt aus den Bewegungsgleichungen unter Zugrundelegung der gemessenen Zustandsgrößen berechnet. Mit diesen Resultaten führt in Abschnitt 4.1 die Überprüfung der Kontinuitätsgleichung für die He-Teilchen zu einer Abschätzung der Teilchendichte der He-Atome und damit des Ionisationsverhältnisses $n_{1} / n_{0}$, das mit dem Ergebnis der statischen Ionisationstheorie verglichen wird. Außerdem wird in Abschnitt 4.2 die Schwerpunktsgeschwindigkeit der zweifach geladenen Ionen, auch unter Benutzung der gemessenen Zustandsgrößen und der Ionisations- und Rekombinationsraten, deren Berechnung im Anhang beschrieben wird, aus der Kontinuitätsgleichung ( 1 a) bestimmt. Wenn die in den Abschnitten 3 und 4 angegebenen unabhängigen Bestimmungen der Schwerpunktsgeschwindigkeit $v_{2 \mathrm{r}}$ zum selben Ergebnis führen, ist per Konsistenz nachgewiesen, daß das Ionisationsverhältnis $n_{2} / n_{1}$ im vorliegenden Plasma durch die Kontinuitätsgleichungen (1) und nicht durch eine statische Ionisationsformel bestimmt ist.

\section{Zur Versuchsanordnung und Erscheinungs- form des Plasmas}

Abb. 1 zeigt schematisch Entladungsgefäß und Magnetfeldspule. Die Anordnung ist rotationssymmetrisch um die strichpunktierte Achse. Der Entladungsstrom zwischen den ringförmigen Aluminiumelektroden wird im Inneren der Spule durch das Magnetfeld und außerhalb der Spule durch Quarztrichter geführt, die mit einer Flußröhre des Magnetfeldes zusammenfallen. Eingesetzte Glas- oder Quarzfenster gestatten end-onBeobachtung. Für side-on-Beobachtung befinden sich Sehschlitze in der Spule. Vor jeder Entladung wird das Entladungsgefäß mit Helium (Einfülldruck 3 Torr) und einem Zusatz von 8\% Kohlenstoff (Propan) gefüllt. Der Entladungsstrom mit einer Stromstärke von $3,8 \cdot 10^{3}$ Amp ist für die Dauer von $700 \mu$ s und das Magnetfeld für die Dauer von 2 ms konstant. Die Anlage zur Erzeugung kurzzeitig konstanter starker Magnetfelder wurde bereits früher beschrieben ${ }^{2}$. Im Inneren der Spule hat das Magnetfeld eine Feldstärke von $4,8 \cdot 10^{4}$ Gauss.

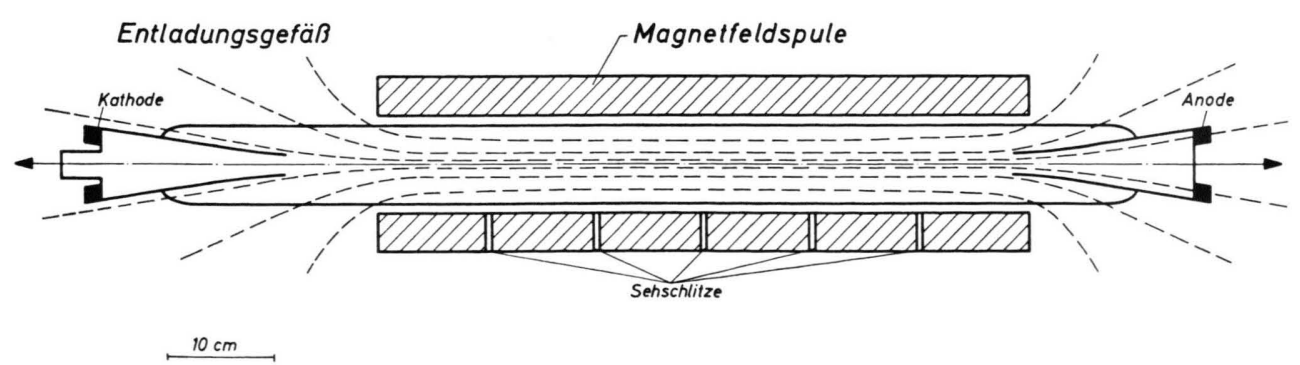

Abb. 1. Entladungsgefäß und Magnetfeldspule mit Magnetfeldkonfiguration.

1 H. WulfF, Proc. VII. Intern. Conf. Phen. Ion. Gases, Belgrade 1, 829 [1965].
2 J. Durand, O. Klüber u. H. Wulff, Z. Angew. Phys. 12, 393 [1960]. 
Side-on aufgenommene Spektren und Drehspiegelbilder im Lichte bestimmter Spektrallinien zeigen, daß das Plasma während der Entladungszeit stationär und in einem scharf gegen das umgebende Neutralgas abgegrenzten Kanal mit einem Durchmesser von etwa $1,7 \mathrm{~cm}$ brennt. Ferner zeigen Aufnahmen, die durch jeweils zwei um einen Azimutwinkel von $90^{\circ}$ gegeneinander versetzte Sehschlitze in der Spule gemacht worden sind, daß das Plasma rotationssymmetrisch ist. Aus Beobachtungen an den verschiedenen Sehschlitzen längs der Spule ist weiter zu entnehmen, daß das Plasma über einen großen Bereich in guter Näherung zylindersymmetrisch ist.

\section{Die Bestimmung der Zustandsgrößen des Plasmas}

\subsection{Elektronentemperatur und Ionisationsverhältnis}

Die Bestimmung der Elektronentemperatur $T_{\mathrm{e}}$ und des Ionisationsverhältnisses $n_{2} / n_{1}$ von zweifach und einfach geladenen Heliumionen erfordert zwei unabhängige Messungen. Dazu werden die Intensitätsverhältnisse je einer He II- und He I-Linie $\left(V_{\mathrm{LL}}\right)$ sowie einer He II-Linie und des Kontinuums bei einer festen Wellenlänge $\left(V_{\mathrm{LK}}\right)$ experimentell ermittelt. Für Elektronentemperaturen $T_{\mathrm{e}}>1,3 \cdot 10^{5}{ }^{\circ} \mathrm{K}$ ist jedoch die Linienstrahlung wegen der vollständigen Ionisation des Heliums praktisch ausgestorben. Daher wird in diesem Bereich $T_{\mathrm{e}}$ aus der Kontinuumsintensität allein bestimmt.

Wie sogleich gezeigt werden wird, darf man die Besetzungsdichten der oberen Zustände von He IIund He I-Linien im vorliegenden Falle aus der SahaBoltzmann-Gleichung berechnen, die z. B. für den He II-Zustand mit der Hauptquantenzahl $p$ und für den durch Quantenzahlen $r$ gekennzeichneten He ITerm die Besetzungsdichten

$n_{1, p}=n_{2} n_{\mathrm{e}} g_{1, p} \frac{h^{3}}{2\left(2 \pi m_{\mathrm{e}} k T_{\mathrm{e}}\right)^{3 / 2}} \exp \left(\frac{\Delta E_{1, p}}{k T_{\mathrm{e}}}\right)$

und

$n_{0, r}=n_{1} n_{\mathrm{e}} \frac{g_{0, r}}{u_{1}} \frac{h^{3}}{2\left(2 \pi m_{\mathrm{e}} k T_{\mathrm{e}}\right)^{3 / 2}} \exp \left(\frac{\Delta E_{0, r}}{k T_{\mathrm{e}}}\right)$

ergibt, wobei $\Delta E_{1, p}$ und $\Delta E_{0, r}$ die Energieabstände von den Ionisationsgrenzen im He II- und He ITermschema und $u_{1}$ die Zustandssumme des einfach geladenen Heliumions bedeuten. Daraus erhält man für die Gesamtintensitäten einer He II-Linie mit dem

3 W. Finkelnburg u. Th. Peters, Handbuch der Physik XXVIII [1957]. oberen Zustand $p$ und einer He I-Linie mit dem oberen Term $r$, die aus einer optisch dünnen Schicht der Dicke $l$ emittiert werden, die Gleichungen

$$
\begin{aligned}
I_{1, p \rightarrow q} & =\frac{h^{4} c g_{1, p} A_{1, p \rightarrow q} l}{8 \pi \lambda_{1, p \rightarrow q}\left(2 \pi m_{\mathrm{e}} k\right)^{3 / 2}} \exp \left(\frac{\left.\Delta E_{1, p}\right)}{k T_{\mathrm{e}}}\right) \frac{n_{2} n_{\mathrm{e}}}{T_{\mathrm{e}}^{3 / 2}} \\
& \equiv b_{1}\left(T_{\mathrm{e}}\right) \frac{n_{2} n_{\mathrm{e}}}{T_{\mathrm{e}}^{3 / 2}}
\end{aligned}
$$

und

$$
\begin{aligned}
I_{0, r \rightarrow s} & =\frac{h^{4} c g_{0, r} A_{0, r \rightarrow s} l}{8 \pi \lambda_{0, r \rightarrow s} u_{1}\left(2 \pi m_{\mathrm{e}} k\right)^{3 / 2}} \exp \left(\frac{\Delta E_{0, r}}{k T_{\mathrm{e}}}\right) \frac{n_{1} n_{\mathrm{e}}}{T_{\mathrm{e}}^{3 / 2}} \\
& \equiv b_{0}\left(T_{\mathrm{e}}\right) \frac{n_{1} n_{\mathrm{e}}}{T_{\mathrm{e}}^{3 / 2}} .
\end{aligned}
$$

Zur Intensität $I_{\mathrm{K}}$ des Kontinuums bei einer festen Wellenlänge liefern frei-frei und frei-gebunden Kontinua von einfach und zweifach geladenen Heliumionen Beiträge, die aus den Kramersschen Formeln zu berechnen sind (siehe z. B. Finkelnburg und Peters ${ }^{3}$ ). Danach ergibt sich für $I_{\mathrm{K}}$ eine Gleichung der Form

$$
I_{\mathrm{K}}=\left(n_{1} c\left(T_{\mathrm{e}}\right)+n_{2} d\left(T_{\mathrm{e}}\right)\right) \frac{n_{\mathrm{e}}}{T_{\mathrm{e}}^{1 / 2}} l .
$$

Dann erhält man für die Intensitätsverhältnisse $V_{\mathrm{LL}}$ und $V_{\mathrm{LK}}$, die zur Bestimmung von $n_{2} / n_{1}$ und $T_{\mathrm{e}}$ dienen, die Beziehungen

$$
\begin{gathered}
V_{\mathrm{LL}}=\frac{I_{1, p \rightarrow q}}{I_{0, r \rightarrow s}}=\frac{\lambda_{0, r \rightarrow s} g_{1, p} A_{1, p \rightarrow q}}{\lambda_{1, p \rightarrow q} g_{0, r} A_{0, r \rightarrow s}} \\
\cdot g_{1,1} \exp \left(\frac{\Delta E_{1, p}-\Delta E_{0, r}}{k T_{\mathrm{e}}}\right) \frac{n_{2}}{n_{1}} \equiv a\left(T_{\mathrm{e}}\right) \frac{n_{2}}{n_{1}}
\end{gathered}
$$

und

$$
V_{\mathrm{LK}}=\frac{I_{1, p \rightarrow q}}{I_{\mathrm{K}}}=\frac{b_{1}\left(T_{\mathrm{e}}\right)}{\left(n_{1} / n_{2}\right) c\left(T_{\mathrm{e}}\right)+d\left(T_{\mathrm{e}}\right)} \frac{1}{T_{\mathrm{e}}},
$$

in denen $p$ und $q$ die oberen und unteren Zustände der betrachteten He II-Linien sowie $r$ und $s$ das obere und untere Niveau der He I-Linie bezeichnen. Die Größen $a, b_{1}, c$ und $d$ in den Gln. (9) und (10) sind so beschaffen, daß sie im betrachteten Elektronentemperaturbereich nur sehr schwach von $T_{\mathrm{e}}$ abhängen. Dann ist zunächst Gl. (9) eine Bestimmungsgleichung für $n_{2} / n_{1}$ bei ungefährer Kenntnis von $T_{\mathrm{e}}$ und Gl. (10) mit dem so gewonnenen $n_{2} / n_{1}$ eine Bestimmungsgleichung für $T_{\mathrm{e}}$. Die Werte von $T_{\mathrm{e}}$ und $n_{2} / n_{1}$ kann man durch Iteration genauer berechnen ${ }^{4}$. - Im Bereich $n_{1} / n_{2}<1$ ist $\left(n_{1} / n_{2}\right) c \ll d$, und $V_{\mathrm{LK}}$ hängt nur noch von $T_{\mathrm{e}}$ ab.

\footnotetext{
4 Ohne eine spezielle statische Ionisationsformel zu benutzen, hat bereits $H$. WULFF ${ }^{1}$ nach diesem Verfahren die Temperatur bestimmt.
} 
Dabei wurde die Zustandssumme

$$
u_{1}=\sum_{p} g_{1, p} \exp \left(-\varepsilon_{1, p} / k T_{\mathrm{e}}\right)
$$

des einfach geladenen Heliumions durch ihr erstes Glied $g_{1,1}=2$ ersetzt. Diese Vereinfachung ist hier erlaubt, weil die Anregungsenergien $\varepsilon_{1, p}$ aller angeregten Zustände $(p>1)$ der wasserstoffähnlichen He-Ionen immer hinreichend groß gegen $k T_{\mathrm{e}}$ sind, wenn diese Ionen merklich im Plasma auftreten. Das bedeutet, daß die Zahl aller einfach geladenen HeIonen praktisch gleich der Zahl der einfach geladenen He-Ionen im Grundzustand ist.

Jetzt soll gezeigt werden, daß man die Besetzungsdichten der oberen Zustände der He II- und He ILinien für das betrachtete Plasma aus der SahaBoltzmann-Gleichung (6) berechnen darf. Zunächst geht aus einer Arbeit von Mewe ${ }^{5}$ hervor, daß die Saha-Boltzmann-Gleichung für alle He II- und He IZustände mit Hauptquantenzahlen $p \geqq 4$ im homogenen stationären (d. h. statischen) Plasma mit Elektronendichten von $n_{\mathrm{e}}=10^{16} \mathrm{~cm}^{-3}$ gilt, und zwar sowohl im Falle optisch dünner als auch im Falle optisch dicker Resonanzstrahlung. In einem inhomogenen stationären Plasma weichen dagegen im allgemeinen die Besetzungsdichten von den statischen Werten ab. Sie müssen für ein solches Plasma aus einem System von gekoppelten Kontinuitätsgleichungen berechnet werden. Für einen Zustand $p$ von He II erhält man z. B. die Kontinuitätsgleichung

$$
\sum_{q \neq p} P_{1 . q \rightarrow p} n_{1, q}-n_{1, p} \sum_{q \neq p} Q_{1 . p \rightarrow q}=\operatorname{div}\left(n_{1, p} \boldsymbol{v}_{1 . p}\right) \text {. }
$$

In dieser Gleichung sind $P_{1, q \rightarrow p}$ und $Q_{1, p \rightarrow q}$ die Raten für die Bevölkerungs- und Entvölkerungsprozesse des Zustandes $p$ durch Übergänge von und zu den Zuständen $q$, wobei $q$ gebundene und freie $\mathrm{Zu}$ stände bezeichnen soll. Eine statische Besetzung der angeregten Zustände $p$ liegt dann vor, wenn - in Analogie zu den Bedingungen ( $5 a$ ) und ( $5 b$ ) für eine statische Ionisation - die Bedingungen

$$
\frac{\left|\operatorname{div}\left(n_{1, p} \boldsymbol{v}_{1, p}\right)\right|}{n_{1, p} \underset{q \neq p}{\sum} Q_{1, p} \rightarrow q} \ll 1
$$

gelten. Die durch $\tau_{1,2 \rightarrow q}=1 / \underset{q \neq 2}{\sum} Q_{1,2 \rightarrow q}$ definierte Zerfallszeit des ersten angeregten Zustandes unterscheidet sich im Falle optisch dünner und optisch

` R. Mewe, Brit. J. Appl. Phys. 18, 107 [1967]. dicker Lyman- $\alpha$-Linie. Im optisch dünnen Falle ist die Zerfallszeit wesentlich durch die Übergangswahrscheinlichkeit $A_{1,2 \rightarrow 1}$ bestimmt. In einem für die Resonanzlinie optisch dicken Plasma, wie es im betrachteten Radiusbereich vorliegt, ist die Zerfallszeit erheblich verlängert, weil der Strahlungszerfall durch die Reabsorption der Resonanzlinie teilweise kompensiert wird. Das drückt sich formal so aus, daß statt $A_{1,2 \rightarrow 1}$ in $\sum_{q \neq 2} Q_{1,2 \rightarrow q}-$ wie im Anhang gezeigt wird $-g \cdot A_{1,2 \rightarrow 1}$ auftritt, wobei $0<g \ll 1$ ist. Selbst in diesem Falle ist die Zerfallszeit so kurz, daß die Bedingung (12) für alle erdenklichen Geschwindigkeits- und Dichteverteilungen im vorliegenden Plasma gilt. Für alle höher angeregten Zustände $p \geqq 3$ ist diese Bedingung ebenfalls erfüllt, und zwar sowohl im Falle optisch dünner als auch optisch dicker Lyman-Serie. (Bekanntlich verlieren mit zunehmender Hauptquantenzahl die Strahlungsprozesse gegenüber den Stoßprozessen schnell an Bedeutung.) Für den Grundzustand $(p=1)$ dagegen gilt diese Bedingung nicht. Das bedeutet, daß in der Kontinuitätsgleichung (11) für den Zustand $p$ der Term $P_{1,1 \rightarrow p} n_{1,1}$ noch von $\operatorname{div}\left(n_{1,1} \boldsymbol{v}_{1,1}\right)$ abhängt. Die dadurch hervorgerufenen Abweichungen von der statischen Besetzung des Zustandes $p$ können nun aber abgeschätzt werden. Nach BATES und Kingston ${ }^{6}$ läßt sich nämlich die Besetzung eines angeregten Zustandes $p$ durch einen Ausdruck der Form

$$
\varrho(p)=r_{0}(p)+r_{1}(p) \varrho(1)+r_{2}(p) \varrho(2)
$$

angeben. Dabei bedeuten $\varrho(p), \varrho(2)$ und $\varrho(1)$ das Verhältnis der tatsächlichen Besetzung des Zustandes $p$, des ersten angeregten Zustandes und des Grundzustandes zur Saha-Boltzmann-Besetzung, während die Größen $r_{0}(p), r_{1}(p)$ und $r_{2}(p)$ für einen bestimmten Wert von $p$ nur von Elektronendichte und -temperatur sowie von atomaren Konstanten abhängen. Wenn - wie es soeben für den vorliegenden Fall gezeigt wurde - die Besetzungsdichte des ersten angeregten Zustandes aus einer statischen Beziehung [vgl. Anhang Gl. (A, 2) ] berechnet werden darf, kann man die obige Gl. (13) in der Form

$$
\varrho(p)=R_{0}(p)+R_{1}(p) \varrho(1)
$$

angeben, wobei $R_{0}(p)$ und $R_{1}(p)$ wiederum für ein festes $p$ nur von Elektronendichte und -temperatur abhängige Koeffizienten sind, die von BATES und

${ }^{6}$ D. R. Bates u. A. E. Kingston, Planet. Space Sci. 11, 1 [1963]. 
Kingston ${ }^{6}$ zunächst für Wasserstoff berechnet wurden. Diese Werte können jedoch unter Benutzung der von Bates, Kingston und McWhirter ${ }^{7}$ angegebenen Skalengesetze in guter Näherung auf den Fall wasserstoffähnlicher Ionen, also auch He II, angewendet werden.

Es ergibt sich, daß im statischen Plasma für $p \geqq 4$ sowohl im Falle optisch dünner als auch komplett optisch dicker Lyman-Strahlung $R_{0}(p) \gg R_{1}(p) \cdot Q(1)$ gilt (vgl. Bates und Kingston ${ }^{6}$ sowie Bates, KingSTON und McWhirTer ${ }^{7}$ ). Somit kann eine Modifikation der Grundzustandsbesetzung $\varrho(1)$ durch Berücksichtigung des Termes $\operatorname{div}\left(n_{1,1} \boldsymbol{v}_{1,1}\right)$ die Besetzung $\varrho(p)$ dieser angeregten Zustände relativ zur Saha-Boltzmann-Gleichung nicht wesentlich beeinflussen. Damit ist bewiesen, daß die Saha-Boltzmann-Gleichung hier für He II-Zustände mit $p \geqq 4$ anwendbar ist.

Auf analoge Weise kann man unter Zugrundelegung der von DRAWIN ${ }^{8}$ für He I-Zustände berechneten Relaxationszeiten zeigen, daß auch die Besetzungsdichten angeregter He I-Niveaus hier aus der Saha-Boltzmann-Gleichung berechnet werden dürfen, wenn die Hauptquantenzahl $p \geqq 4$ ist.

Dagegen ist das Ergebnis der vorliegenden $\mathrm{Ar}$ beit, daß das Ionisationsverhältnis $n_{2} / n_{1}$ von zweifach und einfach geladenen Ionen nicht aus einer statischen Ionisationformel (z. B. der Saha-Gleichung) berechnet werden darf, weil die Bedingungen $(5 \mathrm{a})$ und $(5 \mathrm{~b})$ im betrachteten Plasma nicht gültig sind. Das bedeutet also, daß dieses Verhältnis sich statt aus einer statischen Ionisationsbeziehung [siehe Gl. (4)] aus den Kontinuitätsgleichungen ( $1 \mathrm{a}$ ) und ( $1 \mathrm{~b})$ bestimmt. Dieses Verhältnis wird daher hier experimentell aus dem Intensitätsverhältnis $V_{\mathrm{LL}}$ aus Gl. (9) bestimmt.

In Achsenumgebung $\left(r<0,2 \mathrm{~cm}, T_{\mathrm{e}}>1,3 \cdot 10^{5}{ }^{\circ} \mathrm{K}\right)$ ist das Helium vollständig ionisiert. Dann ergibt sich wegen der Quasineutralität des Plasmas $\left(n_{\mathrm{e}}=2 n_{2}\right)$ aus Gl. (8) für die Kontinuumsintensität

$$
I_{\mathrm{K}}=\frac{1}{2} n_{\mathrm{e}}{ }^{2} / T_{\mathrm{e}}^{1 / 2} \cdot l d\left(T_{\mathrm{e}}\right) .
$$

Mit dem in diesem Bereich aus der Verbreiterung einer CIV-Linie (Abschnitt 2.2) erhaltenen Verlauf von $n_{\mathrm{e}}$ und dem gemessenen Verlauf von $I_{\mathrm{K}}$ erhält man hier die Elektronentemperatur (Abb. 2 a).

7 D. R. Bates, A. E. Kingston u. R. W. P. McWhirter, Proc. Roy. Soc. London A 267, 297 [1962]; 270, 155 [1962].

\subsection{Elektronendichte}

Der radiale Verlauf der Elektronendichte bei kleinen Achsenabständen $(r \leqq 0,6 \mathrm{~cm})$ wird aus dem end-on gemessenen radialen Verlauf der Halbwertsbreite der CIV-Linie $\lambda=2530 \AA \quad\left(5^{2} \mathrm{G} \rightarrow 4{ }^{2} \mathrm{~F}^{0}\right)$ ermittelt. Die Halbwertsbreite dieser nur aus dem zylindersymmetrischen Plasmabereich emittierten Linie ist bei den in Frage kommenden Elektronendichten diesen direkt proportional. Es ist jedoch nicht bekannt, wie groß der absolute Wert der Elektronendichte bei einer bestimmten Halbwertsbreite der C IV-Linie ist, so daß diese Messung nur den relativen Verlauf der Elektronendichte im achsennahen Bereich ergeben kann. Bei größeren Achsenabständen wurden aus den Gln. (9) und (10) das Ionisationsverhältnis $n_{2} / n_{1}$ und die Elektronentemperatur $T_{\mathrm{e}}$ bestimmt. Die zusätzlichen Gleichungen für die Gesamtintensität einer He II-Linie [Gl. (7a)] oder die Kontinuumsintensität [Gl. (8) ] enthalten neben $n_{\mathrm{e}}$ und $T_{\mathrm{e}}$ noch die Ionendichten $n_{1}$ und $n_{2}$. Diese können aber mit der Quasineutralitätsbeziehung $n_{\mathrm{e}}=n_{1}+2 n_{2}$ als Funktionen von $n_{\mathrm{e}}$ und $n_{2} / n_{1}$ ausgedrückt werden. Man erhält dann

$$
n_{2}=\frac{n_{\mathrm{e}}}{1+2 \cdot n_{2} / n_{1}} \cdot \frac{n_{2}}{n_{1}} \quad \text { und } \quad n_{1}=\frac{n_{\mathrm{e}}}{1+2 \cdot n_{2} / n_{1}},
$$

so daß die Intensitäten nur noch von $T_{\mathrm{e}}, n_{2} / n_{1}$ und $n_{\mathrm{e}}$ abhängen. Man kann somit $n_{\mathrm{e}}(r)$ aus dem radialen Verlauf der Linienintensität oder der Kontinuumsintensität gewinnen. Auf eine absolute Bestimmung dieser Intensitäten wurde jedoch wegen der damit verbundenen meßtechnischen Probleme verzichtet, so daß auch in diesem Bereich zunächst nur der relative Verlauf der Elektronendichte ermittelt ist. Statt dessen wurden Absolutwerte durch Messung der Linienprofile von He II- und He ILinien bestimmt, deren Verbreiterungen in Abhängigkeit von der Elektronendichte aus Theorie und Experiment bekannt sind. Da die Spektrallinien von He II und He I aus verschiedenen Zonen des Plasmas emittiert werden, ergibt sich aus der Verbreiterung dieser Linien die absolute Elektronendichte bei zwei verschiedenen Achsenabständen, so daß jetzt das vollständige Elektronendichteprofil bestimmt ist (Abb. 2 b). Die Linienprofile aller hier betrachteten Linien wurden bereits früher im Detail diskutiert ${ }^{9}$.

8 H. W. Drawin, Z. Naturforsch. 19 a, 451 [1964].

9 K. Bergstedt, IPP 3/64 [1967]. 

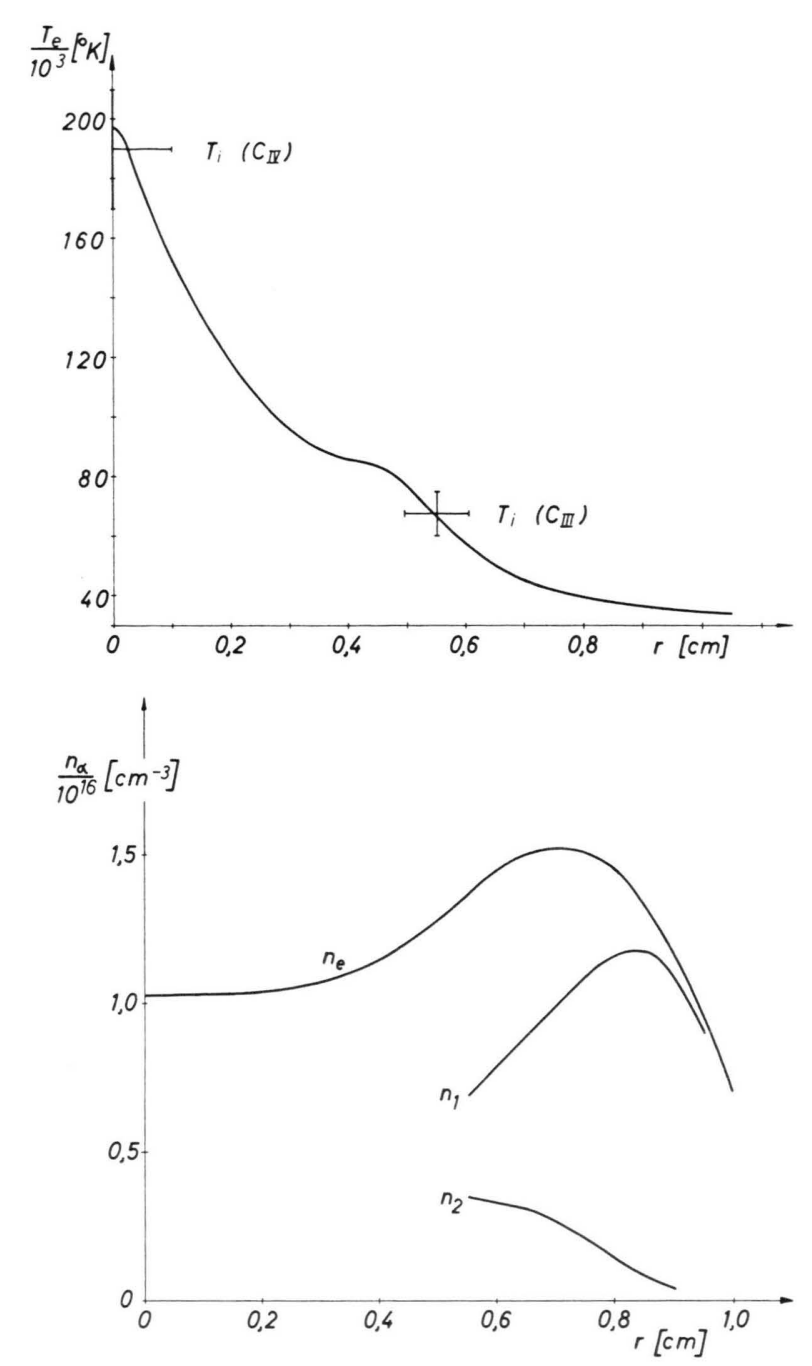

Abb. 2. a) Radialer Elektronentemperaturverlauf $T_{\mathrm{e}}$ und aus den Doppler-Breiten von C IV- und C III-Linien bestimmte Ionentemperaturen $T_{\mathrm{i}}$. b) Radialer Verlauf der Teilchendichten $n_{\alpha}$ von Elektronen $\left(n_{\mathrm{e}}\right)$ und einfach $\left(n_{1}\right)$ und zweifach $\left(n_{2}\right)$ geladenen Heliumionen.

\subsection{Partialdrucke und Gesamtdruck}

Zur Berechnung der Partialdrucke $p_{a}=n_{a} k T_{a}$ der Elektronen $(\alpha=\mathrm{e})$ und Ionen $(\alpha=1$ bzw. $\alpha=2)$ muß neben den bereits bestimmten Dichten und der Elektronentemperatur noch die Ionentemperatur bekannt sein. Diese kann durch Messung der DopplerBreite von dafür geeigneten C IV- und C III-Linien bei zwei Achsenabständen gewonnen werden.

Die Inspektion des Termschemas von C IV zeigt, daß von allen im sichtbaren und Quarz-UV-Spektralbereich liegenden C IV-Linien nur die Feinstrukturkomponenten $\lambda=5801 \AA \quad\left(3{ }^{2} \mathrm{P}_{3 / 2}^{0} \rightarrow 3{ }^{2} \mathrm{~S}_{1 / 2}\right)$ und $\lambda=5812 \AA$

$\left(3{ }^{2} \mathrm{P}_{1 / 2}^{0} \rightarrow 3{ }^{2} \mathrm{~S}_{1 / 2}\right)$ bei der vorliegenden Elektronendichte eine Druckverbreiterung aufweisen, die gegenüber der erwarteten Doppler-Verbreiterung (DopplerHalbwertsbreite ca. $0,5 \AA$ ) zu vernachlässigen ist. Andererseits führt der Zeeman-Effekt in einem Magnetfeld von $50 \mathrm{kG}$ zu dem in Abb. 3 dargestellten Aufspaltungsbild dieser Feinstrukturkomponenten. Es ist ersichtlich, daß nur die im Abstand von etwa $2 \AA$ liegenden $\sigma$-Komponenten von $\lambda=5812 \AA$ für die Messung des thermischen Doppler-Effektes geeignet sind. Die Symmetrie der Profile dieser end-on aufgenommenen Zeeman-Komponenten beweist, daß sie aus einem Bereich praktisch konstanten Magnetfeldes emittiert werden, in dem das Plasma zylindersymmetrisch ist.

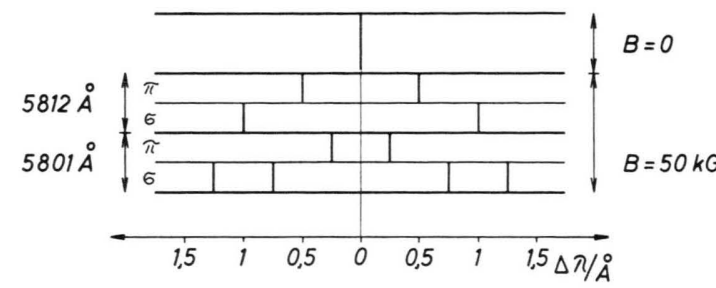

Abb. 3. Aufspaltung der Feinstrukturkomponenten $\lambda=5812 \AA$ $\left(3{ }^{2} \mathrm{P}_{1 / 2}^{0} \rightarrow 3{ }^{2} \mathrm{~S}_{1 / 2}\right)$ und $\lambda=5801 \AA \quad\left(3^{2} \mathrm{P}_{3 / 2}^{0} \rightarrow 3{ }^{2} \mathrm{~S}_{1 / 2}\right)$ der C IV-Linie $3^{2} \mathrm{P}^{0} \rightarrow 3^{2} \mathrm{~S}$ in einem Magnetfeld von $50 \mathrm{kG}$. Oben feldfreie Lage, Mitte $\lambda=5812 \AA$ und unten $\lambda=5801 \AA$ getrennt nach $\sigma$ - und $\pi$-Komponenten.

Außerdem kann an der C III-Linie $\lambda=5696 \AA$ $\left(3^{1} \mathrm{D} \rightarrow 3{ }^{1} \mathrm{P}^{0}\right)$ die Doppler-Breite der $\sigma$-Komponenten gemessen und so die Ionentemperatur in einem Achsenabstand von etwa $0,6 \mathrm{~cm}$ ermittelt werden.

In beiden Fällen findet man im Rahmen der Meßgenauigkeit Übereinstimmung von Ionen- und Elektronentemperatur ${ }^{10}$. Daher sind die Partialdrucke der Elektronen und Ionen mit derselben Temperatur $T_{\alpha}=T$ zu berechnen. Der Gesamtdruck, der auf der Achse einen Wert von etwa einer halben Atmosphäre erreicht, ergibt sich dann als Summe der Partialdrucke.

\section{Die Berechnung der radialen Schwerpunktsgeschwindigkeiten der Ionen aus den Bewegungsgleichungen}

Diesem relativ hohen Achsenwert von einer halben Atmosphäre stehen Druckwerte von rund einer zehntel Atmosphäre vor den Elektroden gegenüber. Infolge dieser Druckdifferenz muß das Plasma axial ausströmen. Den Nachweis für diese Strömung lieferte die end-on beobachtete Doppler-Verschiebung

10 Dabei ist anzumerken, daß wegen der Abhängigkeit $I_{\mathrm{K}}$ $\sim n_{\mathrm{e}}^{2} / T_{\mathrm{e}}^{1 / 2}$ die in der achsennahen Zone aus den gemessenen Verläufen von $I_{\mathrm{K}}$ und $n_{\mathrm{e}}$ erhaltenen Werte von $T_{\mathrm{e}}$ mit relativ großen Fehlern behaftet sein können. 
schmaler Spektrallinien. Da keine zeitliche Abnahme der Dichte im Inneren der Entladung zu bemerken ist, muß das axial ausströmende Plasma durch radial zuströmende Materie ersetzt werden. Eine Überprüfung der Kontinuitätsgleichung für das Gesamtplasma mit der aus der Doppler-Verschiebung erhaltenen axialen Ausströmgeschwindigkeit läßt radiale Schwerpunktsgeschwindigkeiten $v_{\mathrm{r}}$ der Größenordnung $10^{3} \mathrm{~cm} / \mathrm{s}$ in einem Achsenabstand von $r=0,6 \mathrm{~cm}$ erwarten. Neben der mit Sicherheit vorhandenen ambipolaren Diffusion der verschiedenen Plasmakomponenten trägt dann die Plasmaströmung zu den Schwerpunktsgeschwindigkeiten $v_{1 \mathrm{r}}$ und $v_{2 \mathrm{r}}$ der einfach und zweifach geladenen Ionen bei.

Diese radialen Schwerpunktsgeschwindigkeiten werden jetzt unter Benutzung der gemessenen $\mathrm{Zu}$ standsgrößen aus den Bewegungsgleichungen der Ionen und Elektronen berechnet.

Die sich aus der Boltzmann-Gleichung ergebende Bewegungsgleichung einer Plasmakomponente $\alpha$ lautet: $m_{a} n_{\alpha} \frac{\mathrm{d} \boldsymbol{v}_{\alpha}}{\mathrm{d} t}-\boldsymbol{R}_{\alpha}=Z_{\alpha}$ e $n_{\alpha}\left(\boldsymbol{E}+\frac{\boldsymbol{v}_{\alpha}}{c} \times \boldsymbol{B}\right)-\nabla \mathbf{P}_{\alpha}$.

Bei Vernachlässigung der inneren Reibung der Teilchensorte $\alpha$ kann der Drucktensor $\mathrm{P}_{\alpha}$ in dieser Gleichung durch den Partialdruck $p_{a}$ ersetzt werden. Eine Abschätzung der durch den Term $m_{a} n_{a} \mathrm{~d} \boldsymbol{v}_{a} / \mathrm{d} t$ gegebenen Trägheitskräfte in azimutaler und radialer Richtung ergibt, daß sie, verglichen mit den Lorentz-Kräften und den Gradienten der experimentell bestimmten Partialdrucke, vernachlässigt werden dürfen, wenn die auftretenden Geschwindigkeiten klein verglichen mit der Schallgeschwindigkeit des Plasmas sind. Die Reibungskräfte $\boldsymbol{R}_{\alpha}$ auf die Teilchensorte $\alpha$ in einem inhomogenen Plasma im Magnetfeld wurden von verschiedenen Autoren ${ }^{11}$ für das Zweikomponentenplasma sowie von Feneberg ${ }^{12}$ für das Dreikomponentenplasma berechnet.

Wie üblich werden die Schwerpunktsgeschwindigkeiten $\boldsymbol{v}_{\alpha}$ der Elektronen $(\alpha=\mathrm{e})$ und der einfach $(\alpha=1)$ und zweifach $(\alpha=2)$ geladenen Ionen durch Linearkombinationen von Schwerpunktsgeschwindigkeit $\boldsymbol{v}$ des Plasmas, Stromdichte $\boldsymbol{j}$ und einer Diffusionsgröße $\boldsymbol{d}$ ausgedrückt. Diese Größen sind durch die folgenden Beziehungen definiert:

$$
\begin{gathered}
\boldsymbol{v}=\frac{m_{\mathrm{He}}\left(n_{1} \boldsymbol{v}_{1}+n_{2} \boldsymbol{v}_{2}\right)+m_{\mathrm{e}} n_{\mathrm{e}} \boldsymbol{v}_{\mathrm{e}}}{m_{\mathrm{He}}\left(n_{1}+n_{2}\right)+m_{\mathrm{e}} n_{\mathrm{e}}}, \\
\boldsymbol{j} / e=n_{1} \boldsymbol{v}_{1}+2 n_{2} \boldsymbol{v}_{\mathbf{2}}-n_{\mathrm{e}} \boldsymbol{v}_{\mathrm{e}}, \\
\boldsymbol{d}=\frac{n_{1} n_{2}}{\left(n_{1}+n_{2}\right) n_{\mathrm{e}}}\left(\boldsymbol{v}_{2}-\boldsymbol{v}_{1}\right) .
\end{gathered}
$$

11 Zum Beispiel: S. I. Braginskir, Soviet Phys.-JETP 6, 358 [1958]. - W. Feneberg u. H. Fisser, Proc. VII. Intern. Conf. Phen. Ion. Gases, Belgrade 2, 63 [1965].
Aus diesen Gleichungen erhält man für die Schwerpunktsgeschwindigkeiten der Elektronen und Ionen

$$
\begin{gathered}
\boldsymbol{v}_{e}=\boldsymbol{v}-\frac{n_{1}+n_{2} \boldsymbol{j}}{A n_{\mathrm{e}}}+\frac{n_{1}+n_{2}}{A} \boldsymbol{d}, \\
\boldsymbol{v}_{1}=\boldsymbol{v}+\frac{\beta}{A} \frac{\boldsymbol{j}}{e}-\frac{n_{\mathrm{e}}\left(n_{1}+n_{2}\right)}{n_{1} A}(1+2 \beta) \boldsymbol{d}, \\
\boldsymbol{v}_{2}=\boldsymbol{v}+\frac{\beta}{A} \frac{\boldsymbol{j}}{e}+\frac{n_{\mathrm{e}}\left(n_{1}+n_{2}\right)}{n_{2} A}(1+\beta) \boldsymbol{d} .
\end{gathered}
$$

Dabei sind $\beta=m_{\mathrm{e}} / m_{\mathrm{He}} \ll 1$ und $A=n_{1}+n_{2}+\beta n_{\mathrm{e}}$ gesetzt. Mit $\beta=0$ und der Quasineutralitätsbeziehung $n_{\mathrm{e}}=n_{1}+2 n_{2}$ sowie - den Versuchsbedingungen entsprechend - mit $j_{\mathrm{r}}=0$ ergeben sich daraus die radialen Schwerpunktsgeschwindigkeiten

$$
\begin{aligned}
& v_{\mathrm{er}}=v_{\mathrm{r}}+d_{\mathrm{r}}, \\
& v_{1 \mathrm{r}}=v_{\mathrm{r}}-\left(n_{\mathrm{e}} / n_{1}\right) d_{\mathrm{r}}, \\
& v_{2 \mathrm{r}}=v_{\mathrm{r}}+\left(n_{\mathrm{e}} / n_{2}\right) d_{\mathrm{r}} .
\end{aligned}
$$

Man hat jetzt die Komponentengeschwindigkeiten $\boldsymbol{v}_{\mathrm{e}}$, $\boldsymbol{v}_{1}$ und $\boldsymbol{v}_{2}$ aus den Gln. $(19 \mathrm{a}-\mathrm{c})$ in die Bewegungsgleichungen einzusetzen. Wegen der Zylindersymmetrie des Plasmas und den folgenden, hier gültigen Voraussetzungen ergeben sich dabei erhebliche Vereinfachungen. Die praktisch gleichbedeutenden Voraussetzungen $v_{\mathrm{e} z} B_{\varphi} \ll v_{\mathrm{e} \varphi} B_{z}$ und $j_{z} B_{\varphi} \ll j_{\varphi} B_{z}$ besagen, da $\beta$ in der Bewegungsgleichung des Gesamtplasmas

$$
\frac{\partial p}{\partial r}=\frac{1}{c}\left(j_{\varphi} B_{z}-j_{z} \mathbf{B}_{\varphi}\right)
$$

der Anteil des Pinch-Effektes am radialen Druckaufbau des Plasmas vernachlässigt wird. Die Gültigkeit dieser Voraussetzung für das betrachtete Plasma wurde von KlÜBER ${ }^{13}$ und GrassmanN ${ }^{14}$ nachgewiesen. Die weitere Voraussetzung $v_{2 z} B_{\varphi} \ll v_{2 p} B_{z}$ gilt, weil die Beobachtungen der Doppler-Verschiebung von Spektrallinien am Spulenausgang ergeben haben, daß die Plasmaabströmung nur in unmittelbarer Achsennähe stattfindet. Außerdem erfolgt diese Abströmung sowohl in Richtung zur Anode als auch zur Kathode, so daß an einer Stelle $z^{\prime}$ innerhalb der Spule die Abströmgeschwindigkeit $v_{z}\left(z^{\prime}\right)$ sogar auf der Achse verschwindet. Dann ist für die jetzt betrachteten relativ großen Achsenabstände im Mittelteil der Entladung sicher $v_{2 z} B_{\varphi} \ll v_{2 \varphi} B_{z}$.

Die $r$ - und $\varphi$-Komponenten der Bewegungsgleichungen bilden zusammen mit der Gleichung $j_{r}=0$ ein System von sieben gekoppelten Gleichungen, die die elektrische Feldstärke $E_{\mathrm{r}}$ und die $r$ - und $\varphi$-Komponenten von $\boldsymbol{v}, \boldsymbol{j}$ und $\boldsymbol{d}$ als unbekannte Größen enthalten. Aus diesem Gleichungssystem kann man durch elementare Rechnung unter Benutzung der gemessenen Zustandsgrößen $v_{\mathrm{r}}$ und $d_{\mathrm{r}}$ bestimmen. Die Schwerpunktsgeschwindigkeiten der Ionen ergeben sich dann aus den Gln. $(20 \mathrm{~b})$ und $(20 \mathrm{c})$.

12 W. Feneberg, Veröffentlichung in Vorbereitung.

13 O. KLÜBER, Z. Naturforsch. 22 a, 1599 [1967].

14 P. H. Grassmann, Z. Naturforsch. 23 a, 251 [1968]. 
Sie sind in Tab. 1, nach den Anteilen der Schwerpunktsgeschwindigkeit $v_{\mathrm{r}}$ des Plasmas und den ambipolaren Diffusionsgeschwindigkeiten $-\left(n_{\mathrm{e}} / n_{1}\right) d_{\mathrm{r}}$ und $\left(n_{\mathrm{e}} / n_{2}\right) d_{\mathrm{r}}$ der einfach und zweifach geladenen Ionen aufgegliedert, angegeben.

\begin{tabular}{|c|c|c|c|c|c|c|}
\hline$r$ & $v_{\mathrm{r}}$ & $-\frac{n_{\mathrm{e}}}{n_{1}} d_{\mathrm{r}}$ & $v_{1 \mathrm{r}}$ & $\frac{n_{\mathrm{e}}}{n_{2}} d_{\mathrm{r}}$ & $v_{2 \mathrm{r}}$ & $\left(n_{1}+n_{2}\right) v_{\mathrm{r}} r$ \\
\hline$(\mathrm{cm})$ & \multicolumn{5}{|c|}{$\left(10^{3} \mathrm{~cm} / \mathrm{s}\right)$} & $\left(10^{19} / \mathrm{cm} \cdot \mathrm{s}\right)$ \\
\hline 0,55 & $-3,0$ & $-6,2$ & $-9,2$ & 12 & 9 & $-1,6$ \\
\hline 0,6 & $-2,5$ & $-3,4$ & $-5,9$ & 8,4 & 5,9 & $-1,7$ \\
\hline 0.65 & $-2,2$ & $-2,6$ & $-4,8$ & 7,4 & 5,2 & $-1,6$ \\
\hline 0,7 & $-1,8$ & $-1,8$ & $-3,6$ & 6,6 & 4,8 & $-1,2$ \\
\hline 0,75 & $-1,6$ & & & 6,1 & 4.5 & $-0,8$ \\
\hline
\end{tabular}

Tab. 1. Die radialen Schwerpunktsgeschwindigkeiten $v_{1 \mathrm{r}}$ und $v_{2 r}$ der einfach und zweifach geladenen Ionen und ihre Aufgliederung in die Anteile $v_{\mathrm{r}}$ der Schwerpunktsströmung und - $\left(n_{\mathrm{e}} / n_{1}\right) d_{\mathrm{r}}$ und $\left(n_{\mathrm{e}} / n_{2}\right) d_{\mathrm{r}}$ der ambipolaren Diffusion sowie das in Gl. (22) auftretende Produkt $\left(n_{1}+n_{2}\right) v_{\mathrm{r}} r$ für verschiedene Achsenabstände $r$.

\section{Diskussion der Resultate}

\subsection{Ionisationsverhältnis $n_{1} / n_{0}$}

In den Beziehungen

$$
\operatorname{div}\left(n_{\alpha} \boldsymbol{v}_{\alpha}\right)=\frac{1}{r} \frac{\partial\left(n_{\alpha} v_{\alpha r} r\right)}{\partial r}+\frac{\partial\left(n_{\alpha} v_{\alpha z}\right)}{\partial z}
$$

für die einfach $(\alpha=1)$ und zweifach $(\alpha=2)$ geladenen Ionen lassen sich die Terme

$$
\frac{1}{r} \frac{\partial\left(n_{\alpha} v_{\alpha r} r\right)}{\partial r}
$$

mit den Ergebnissen in Tab. 1 ermitteln. Diesen Termen gegenüber dürfen die Terme $\partial\left(n_{\alpha} v_{\alpha z}\right) / \partial z$ vernachlässigt werden, weil einerseits die achsenparallele Ausströmungsgeschwindigkeit des Plasmas - wie es bereits erörtert wurde - bei den betrachteten großen Achsenabständen nur verhältnismäßig kleine Werte annimmt und andererseits die für deren Änderung in $z$-Richtung charakteristischen Längen groß sind, so daß $\partial\left(n_{a} v_{a z}\right) / \partial z$ relativ klein ist. Außerdem rechtfertigt die Konsistenz der in dieser Arbeit erhaltenen Ergebnisse diese Vernachlässigung. Dann erhält man durch Addition der Kontinuitätsgleichungen $(1 \mathrm{a})$ und $(1 \mathrm{~b})$ für die einfach und zweifach geladenen Ionen $(Z=2)$ die Kontinuitätsgleichung

$$
1 \frac{\partial\left[r\left(n_{1} v_{1 r}+n_{2} v_{2 r}\right)\right]}{\partial r}=0
$$

für alle Heliumionen. Aus dieser Gleichung ergibt sich unter Berücksichtigung von Gl. (16) mit $m_{\mathrm{e}} / m_{\mathrm{He}} \ll 1$ und $j_{\mathrm{r}}=0$ die Beziehung

$$
\left(n_{1}+n_{2}\right) v_{\mathrm{r}} r=\text { const . }
$$

Diese Kontinuitätsgleichung für die Heliumionen gilt nach Tab. 1 im Innern der Entladung. Man erhält hier $\left(n_{1}+n_{2}\right) v_{\mathrm{r}} r=-1,65 \cdot 10^{19} / \mathrm{cm} \cdot \mathrm{s}$. Bei gröBeren Achsenabständen findet man dagegen kleinere Werte. Diese Abweichungen treten auf, weil in Gl. (22) die Neutralteilchen nicht berücksichtigt sind. An Stelle dieser Gleichung gilt hier die Kontinuitätsgleichung

$$
\left(n_{0}+n_{1}+n_{2}\right) v_{\mathrm{r}} r=\mathrm{const}
$$

unter Einschluß der Neutralteilchen. Ihre Dichte $n_{0}$ ist weder aus den Messungen noch aus der Rechnung zu entnehmen. Sie kann jedoch mit Hilfe der Gl. (23) abgeschätzt werden. Die auf Grund dieser Abschätzung resultierenden Verhältnisse $\left(n_{1} / n_{0}\right)$ Exp der Dichten von einfach geladenen und neutralen Heliumteilchen sind zusammen mit den aus der Koronagleichung ${ }^{15}$ berechneten Verhältnissen $\left(n_{1} / n_{0}\right)^{\prime}$ in Tab. 2 angegeben.

\begin{tabular}{cccc}
\hline$r \mathrm{~cm}$ & 0,75 & 0,8 & 0,85 \\
$\left(n_{1} / n_{0}\right)_{\operatorname{Exp}}$ & 12 & 5 & 2 \\
$\left(n_{1} / n_{0}\right)^{\prime}$ & 126 & 95 & 67 \\
\hline
\end{tabular}

Tab. 2. Die aus der Kontinuitätsgleichung (23) des Plasmas abgeschätzten Ionisationsverhältnisse $\left(n_{1} / n_{0}\right)$ Exp von einfach geladenen und neutralen Heliumteilchen und die aus der Koronaformel berechneten statischen Verhältnisse $\left(n_{1} / n_{0}\right)^{\prime}$ für verschiedene Achsenabstände $r$.

Das sich aus der statischen Ionisationsbeziehung

$$
J_{0} n_{0}-R_{1} n_{1}=0
$$

- mit den für dieses spezielle Plasma nicht bekannten Ionisations- und Rekombinationsraten $J_{0}$ und $R_{1}$ - ergebende Ionisationsverhältnis $n_{1} / n_{0}=J_{0} / R_{1}$ ist sicher größer als das aus der Koronagleichung berechnete Verhältnis $\left(n_{1} / n_{0}\right)^{\prime}$, weil die Koronagleichung von allen statischen Ionisationsformeln (z. B. auch der Saha-Gleichung) das kleinste Verhältnis $n_{1} / n_{0}$ liefert. Da das hier ermittelte Verhältnis $\left(n_{1} / n_{0}\right)_{\text {Exp }}$ aber noch wesentlich kleiner ist, muß geschlossen werden, daß $n_{1} / n_{0}$ im betrachteten Experiment nicht aus der statischen Beziehung (24) berechnet werden darf.

15 Zum Beispiel: A. Unsöld, Physik der Sternatmosphären, Springer-Verlag, Berlin 1955. 


\subsection{Ionisationsverhältnis $n_{2} / n_{1}$}

Das trifft auch für das Ionisationsverhältnis $n_{2} / n_{1}$ der Heliumionen zu. Um das zu zeigen, müssen zunächst die Ionisations- und Rekombinationsraten $J_{1}$ und $R_{2}$ in den Kontinuitätsgleichungen

$$
\begin{array}{ll} 
& R_{2} n_{2}-J_{1} n_{1}=\operatorname{div}\left(n_{1} \boldsymbol{v}_{1}\right) \\
\text { und } & J_{1} n_{1}-R_{2} n_{2}=\operatorname{div}\left(n_{2} \boldsymbol{v}_{2}\right)
\end{array}
$$

berechnet werden. Diese Berechnung wird im Anhang beschrieben. Es ergeben sich für das betrachtete Plasma z. B. in einem Achsenabstand $r=0,7 \mathrm{~cm}$ mit den gemessenen Zustandsgrößen

$$
J_{1} n_{1}=3,2 \cdot 10^{19} / \mathrm{cm}^{3} \cdot \mathrm{sec}
$$$$
\text { und } \quad R_{2} n_{2}=7,0 \cdot 10^{19} / \mathrm{cm}^{3} \cdot \mathrm{sec} \text {. }
$$

Daraus folgt bereits, daß die statische Ionisationsbeziehung

$$
J_{1} n_{1}-R_{2} n_{2}=0
$$

hier nicht gilt. Es ergibt sich vielmehr, daß

$$
J_{1} n_{1}-R_{2} n_{2}=-3,8 \cdot 10^{19} / \mathrm{cm}^{3} \cdot \mathrm{sec}
$$

ist, während in Abschnitt 3 die Bewegungsgleichungen ebenfalls unter Zugrundelegung der gemessenen Zustandsgrößen in überraschend guter Übereinstimmung mit diesem Wert z. B.

$$
\begin{aligned}
J_{1} n_{1}-R_{2} n_{2}=\operatorname{div}\left(n_{2} \boldsymbol{v}_{2}\right) & \approx \frac{1}{r} \frac{\partial\left(n_{2} v_{2 r} r\right)}{\partial r} \\
& =-4,5 \cdot 10^{19} / \mathrm{cm}^{3} \cdot \mathrm{sec}
\end{aligned}
$$

liefern.

Durch Integration der Kontinuitätsgleichung (25 b) kann man dann die Schwerpunktsgeschwindigkeit $v_{2 \mathrm{r}}$ der zweifach geladenen Ionen unabhängig von den aus den Bewegungsgleichungen berechneten Werten bestimmen.

Es ergibt sich

$$
F_{2}(r) \equiv \int_{r}^{r^{*}}\left(J_{1} n_{1}-R_{2} n_{2}\right) r^{\prime} \mathrm{d} r^{\prime}=n_{2}\left(r^{\prime}\right) v_{2 \mathrm{r}}\left(r^{\prime}\right) r_{r}^{r^{*}} \text {. }
$$

Der Achsenabstand $r^{*}$ wird jetzt so groß gewählt, $\mathrm{da} ß$ an dieser Stelle praktisch keine zweifach geladenen Ionen mehr vorhanden sind. Dann kann $n_{2}\left(r^{*}\right) v_{2 \mathrm{r}}\left(r^{*}\right) r^{*}$ gegen $n_{2}(r) v_{2 \mathrm{r}}(r) r$ vernachlässigt werden, so daß $F_{2}$ nur noch vom kleineren Achsenabstand $r$ abhängt, und man erhält

$$
v_{2 \mathrm{r}}=-F_{2}(r) / n_{2}(r) r \text {. }
$$

(Diese Vereinfachung läßt sich für die einfach geladenen Ionen nicht durchführen, weil das Ionisa- tionsverhältnis nur im Bereich $n_{2} / n_{1}<1$ gemessen werden konnte, so daß sich auf diese Weise lediglich die Schwerpunktsgeschwindigkeiten der zweifach geladenen Ionen ermitteln lassen.) Durch graphische Integration von Gl. (27) gewinnt man die in Tab. 3 eingetragenen Werte, die zur Unterscheidung von den ebenfalls angegebenen aus den Bewegungsgleichungen berechneten Resultaten $\left(v_{2 \mathrm{r}}\right)_{\mathrm{B}}$ mit dem In-

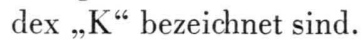

\begin{tabular}{rcllll}
\hline$r$ & $\mathrm{~cm}$ & 0,6 & 0,65 & 0,7 & 0,75 \\
$\left(v_{2 \mathrm{r}}\right)_{\mathrm{K}}$ & $10^{3} \mathrm{~cm} / \mathrm{s}$ & 5 & 4,2 & 4,1 & 3,8 \\
$\left(v_{2 \mathrm{r}}\right)_{\mathrm{B}}$ & $10^{3} \mathrm{~cm} / \mathrm{s}$ & 5,9 & 5,2 & 4,8 & 4,5 \\
\hline
\end{tabular}

Tab. 3. Die aus den Bewegungsgleichungen berechneten und unabhängig davon aus der Kontinuitätsgleichung (25 b) bestimmten radialen Schwerpunktsgeschwindigkeiten $\left(v_{2 r}\right) \mathrm{B}$ und $\left(v_{2}\right) \mathrm{K}$ der zweifach geladenen Heliumionen für verschiedene Achsenabstände $r$.

In Anbetracht der zahlreichen möglichen Fehlerquellen bei den beiden unabhängigen Bestimmungen von $v_{2 \text { 2r }}$ darf die Übereinstimmung der Ergebnisse wohl als sehr gut gelten. Damit ist nachgewiesen, daß das gemessene Ionisationsverhältnis $n_{2} / n_{1}$ hier nicht durch eine statische Ionisationsformel wiedergegeben werden kann (vgl. dazu auch Abb. 5), sondern daß es durch die Kontinuitätsgleichungen (25 a) ${ }^{16}$ und ( $25 \mathrm{~b}$ ) bestimmt ist, die natürlich für den Grenzfall eines statischen Plasmas in die aus Gl. (26) erhaltene statische Ionisationsformel übergehen, und zwar dann, wenn die Bedingungen ( 5 a) und $(5 \mathrm{~b})$ gelten.

Die Bedeutung, welche dieser in der vorliegenden Arbeit aufgezeigte neue Gesichtspunkt bezüglich der Ionisation in einem nichtstatischen Plasma für die spektroskopische Bestimmung der Elektronentemperatur hat, wird in Abb. 4 veranschaulicht. Dazu sind in diesem Diagramm das gemessene, korrekte Elektronentemperaturprofil $\left(T_{\mathrm{e}}\right)_{\mathrm{Exp}}$ und die „Temperaturverläufe" eingezeichnet, die unter Benutzung von statischen Ionisationsformeln aus den gemessenen Intensitätsverhältnissen von Spektrallinien aufeinanderfolgender Ionisationsstufen desselben Elements bestimmt wurden. In allen Fällen wurde dabei vorausgesetzt, daß die Besetzungsdichten der oberen Niveaus der betrachteten Linien aus der Saha-

16 Im Radiusbereich $0,75<r \leqq 0,9 \mathrm{~cm}$, in dem neben den Heliumionen auch Heliumatome in merklicher Konzentration vorhanden sind, gilt für die einfach geladenen Ionen die Kontinuitätsgleichung

$$
R_{2} n_{2}+I_{0} n_{0}-\left(I_{1}+R_{1}\right) n_{1}=\operatorname{div}\left(n_{1} \boldsymbol{v}_{1}\right) .
$$


Boltzmann-Gleichung berechnet werden dürfen. Die dann mit dem gemessenen Intensitätsverhältnis $V_{\mathrm{LL}}$ einer He II- und He I-Linie aus Gl. (9) unter Verwendung verschiedener statischer Beziehungen zur Bestimmung des Ionisationsverhältnisses $n_{2} / n_{1}$ ermittelten "Temperaturverläufe" sind in Abb. 4 angegeben, und zwar wurde das Verhältnis $n_{2} / n_{1}$ aus Gl. (26) mit den für dieses spezielle Plasma im Anhang bestimmten Raten $I_{1}$ und $R_{2}$ sowie aus der Saha-Gleichung und der Koronaformel berechnet.

Außerdem wurde die "Temperatur" mit dem gemessenen Intensitätsverhältnis einer C IV-Linie $\left(\lambda=4658 \AA: 6{ }^{2} \mathrm{H}^{0} \rightarrow 5{ }^{2} \mathrm{G}\right.$ usw. $)$ und einer C IIILinie $\left(\lambda=4187 \AA: 5{ }^{1} \mathrm{G} \rightarrow 4{ }^{1} \mathrm{~F}^{0}\right)$ aus einer zu Gl. (9) analogen Gleichung ermittelt, wobei das Ionisationsverhältnis $n_{4} / n_{3}$ von vierfach und dreifach geladenen Kohlenstoffionen mit den von MAHN ${ }^{17}$ berechneten Ionisations- und Rekombinationsraten $J_{3}$ und $R_{4}$ aus der statischen Beziehung

$$
J_{3} n_{3}-R_{4} n_{4}=0
$$

bestimmt wurde (für Details vgl. ${ }^{9}$ ). Die in diesem Fall auftretenden sehr großen Abweichungen vom korrekten Elektronentemperaturprofil sind möglicherweise nur zum Teil darauf zurückzuführen, daß hier an Stelle der benutzten statischen Beziehung (29) z. B. für die heliumähnlichen C V-Ionen die Kontinuitätsgleichung

$$
J_{3} n_{3}-R_{4} n_{4}=\operatorname{div}\left(n_{4} \boldsymbol{v}_{4}\right)
$$

gilt. Es läßt sich nämlich keinesfalls beweisen, daß in diesem nichtstatischen Plasma die Besetzungsdichten der betrachteten angeregten CIV- und C IIINiveaus aus der Saha-Boltzmann-Gleichung berechnet werden dürfen; denn deren Anwendbarkeit hängt für einen gegebenen Elektronendichte- und Elektronentemperaturbereich selbst in statischen Plasmen entscheidend von der Struktur der im allgemeinen sehr komplizierten Termschemata und von in den meisten Fällen nicht bekannten Größen, wie Stoßquerschnitten und Übergangswahrscheinlichkeiten, ab. (Diese mögliche Fehlerquelle für die Besetzungsdichten angeregter Niveaus aus den relativ einfachen Termschemata von He II und He I konnte dagegen bei der Herleitung von Gl. (9) in Abschnitt 2 sogar für das vorliegende nichtstatische Plasma im Detail diskutiert werden.) Außerdem sind die für die Bestimmung des statischen Verhältnisses $n_{4} / n_{3}$ aus Gl. (29)

17 C. MAHN, Z. Naturforsch. 22 a, 1939 [1967].

18 T. Holstein, Phys. Rev. 72, 1212 [1947]; 83, 1159 [1951]. wichtigen Raten $J_{3}$ und $R_{4}$ sicher nur mit einem bedeutend geringeren Grad von Genauigkeit bekannt als die Raten $J_{1}$ und $R_{2}$ für He II.

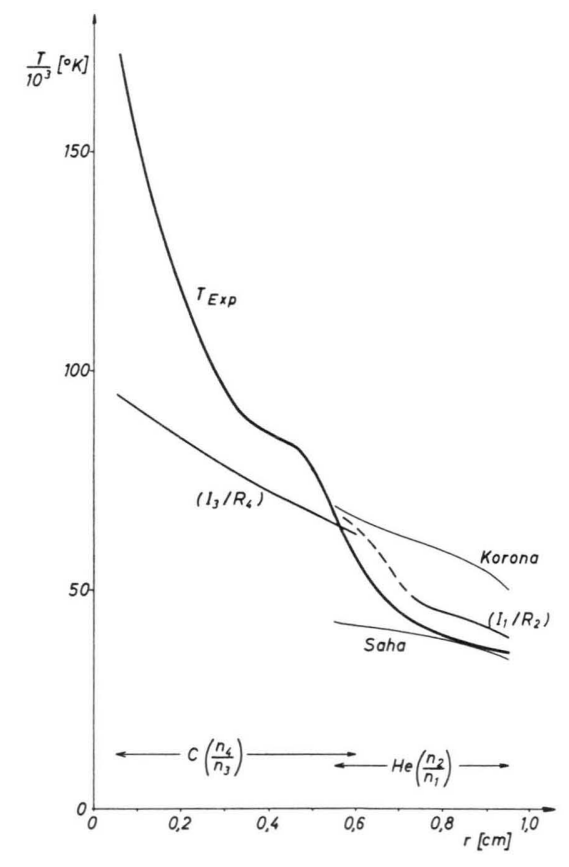

Abb. 4. Vergleich des korrekten, gemessenen radialen Temperaturprofils $T_{\operatorname{Exp}}$ mit den unter Verwendung statischer Ionisationsformeln aus gemessenen Linienintensitätsverhältnissen bestimmten „Temperaturverläufen“. $\mathrm{He}\left(n_{2} / n_{1}\right)$ : „Temperaturverläufe" bestimmt aus dem Intensitätsverhältnis einer He II- und He I-Linie, wobei das statische Ionisationsverhältnis $n_{2} / n_{1}$ aus Gl. (26) mit den speziellen Raten $J_{1}$ und $R_{2}$ für dieses Plasma (Übergang zum optisch dünnen Fall gestrichelt) sowie aus der Saha-Gleichung und der Koronagleichung berechnet ist. C $\left(n_{4} / n_{3}\right)$ : ,Temperaturverlauf, bestimmt aus dem Intensitätsverhältnis einer C IV- und C III-Linie, wobei das statische Ionisationsverhältnis $n_{4} / n_{3}$ aus Gl. (29) mit den von

MAHN ${ }^{17}$ angegebenen Raten $J_{3}$ und $R_{4}$ berechnet ist.

Zum Schluß sei darauf hingewiesen, daß - wie es soeben für die Elektronentemperaturmessung aufgezeigt wurde - Diskrepanzen bei der spektroskopischen Bestimmung von Übergangswahrscheinlichkeiten, Transportkoeffizienten usw. möglicherweise auf die nicht gerechtfertigte Verwendung statischer Ionisationsformeln (vorwiegend der Saha-Gleichung) zurückzuführen sind.

\section{Anhang}

Zur Berechnung der Ionisations-und Rekombinationsraten $J_{1}$ und $R_{2}$

In Abschnitt 2 wurde bereits erläutert, daß durch die Reabsorption der Lyman- $\alpha$-Linie im Plasma die 
Lebensdauer des ersten angeregten He II-Zustandes erheblich verlängert wird. Demnach wird seine Besetzungsdichte durch die Reabsorption dieser Linie wesentlich erhöht. Weil aber der Energieabstand vom Grundzustand zum ersten angeregten $\mathrm{Zu}$ stand $75 \%$ der Ionisierungsenergie $\chi_{1}$ beträgt und ferner im betrachteten Elektronentemperaturbereich $\chi_{1} \gg k T_{\text {e }}$ gilt, wird dieser erste angeregte Zustand von großer Bedeutung bei der stufenweisen Ionisation und Rekombination von He II sein. Daher ergeben sich besonders in den beiden von BATES, KInGSTON und McWhiRTER ${ }^{7}$ betrachteten Grenzfällen optisch dünner und komplett optisch dicker Lyman-Strahlung sowohl für die Ionisationsrate $J_{1}$ als auch für die Rekombinationsrate $R_{2}$ sehr unterschiedliche Werte. Wenn - wie es hier der Fall ist - die emittierten Photonen der Lyman-Serie nur zum Teil im Plasma reabsorbiert werden, kann das nach Holstein ${ }^{18}$ dadurch berücksichtigt werden, daß man in den Bilanzgleichungen (11) für das optisch dünne Plasma die Übergangswahrscheinlichkeiten $A_{1, p \rightarrow 1}$ der Lyman-Linien durch $g(p) A_{1, p \rightarrow 1}$ ersetzt, wobei der „escape factor" $g(p)$ im Intervall $0<g(p)<1$ liegt. Die beiden obigen Grenzfälle sind dann durch $g(p)=1$ (optisch dünn) und $g(p)$ $=0$ (Lyman-Linien komplett optisch dick) gegeben. (Dieser Faktor wird in sehr übersichtlicher Weise auch von McWHIRTER ${ }^{19}$ berechnet.)

Zur Berechnung der Ionisations- und Rekombinationsraten kann man dann ein vereinfachtes Termschema betrachten, wie es von $\mathrm{MEWE}^{5}$ (Fig. 1) eingeführt und durch Vergleich mit den von BATES, Kingston und McWhirTer ${ }^{7}$ für $g(p)=0$ und 1 erhaltenen Ergebnissen gerechtfertigt wurde.

In diesem vereinfachten Modell nehmen im Falle eines inhomogenen, nichtstatischen Plasmas die Kontinuitätsgleichungen der zweifach geladenen Heliumionen und der einfach geladenen Heliumionen im Grundzustand und im ersten angeregten Zustand die Form

$$
\begin{aligned}
& n_{1,1} S_{1 \rightarrow \mathrm{K}}+n_{1,2} S_{2 \rightarrow \mathrm{K}}-n_{2}\left(\alpha_{\mathrm{K} \rightarrow 1}+\alpha_{\mathrm{K} \rightarrow 2}\right)=\operatorname{div}\left(n_{2} \boldsymbol{v}_{2}\right), \\
& n_{2} \alpha_{\mathrm{K} \rightarrow 1}+n_{1,2}\left(S_{2 \rightarrow 1}+g(1) A_{1,2 \rightarrow 1}\right)-n_{1,1}\left(S_{1 \rightarrow 2}+S_{1 \rightarrow \mathrm{K}}\right)=\operatorname{div}\left(n_{1,1} \boldsymbol{v}_{1,1}\right), \\
& n_{2} \alpha_{\mathrm{K} \rightarrow 2}+n_{1,1} S_{1 \rightarrow 2}-n_{1,2}\left(S_{2 \rightarrow 1}+S_{2 \rightarrow \mathrm{K}}+g(1) A_{1,2} \rightarrow 1\right)=\operatorname{div}\left(n_{1,2} \boldsymbol{v}_{1,2}\right)
\end{aligned}
$$

an. Wie bereits in Abschnitt 2 gezeigt wurde, darf im vorliegenden Plasma die Kontinuitätsgleichung $(\mathrm{A}, \mathrm{lc})$ des ersten angeregten He II-Zustandes in ihrer statischen Form [mit $\left.\operatorname{div}\left(n_{1,2} \boldsymbol{v}_{1,2}\right)=0\right]$ benutzt werden. Aus dieser Gleichung ergibt sich dann die Dichte

$$
n_{1,2}=\frac{n_{2} \propto_{\mathrm{K} \rightarrow 2}+n_{1} S_{1 \rightarrow 2}}{S_{2 \rightarrow 1}+S_{2 \rightarrow \mathrm{K}}+g(1) A_{1,2 \rightarrow 1}}
$$

im ersten angeregten Zustand, wobei die Dichte $n_{1,1}$ im Grundzustand durch die Dichte $n_{1}$ aller He IIIonen ersetzt wurde. Nach Einsetzen dieses Ausdruckes in die Beziehungen $(\mathrm{A}, \mathrm{la})$ und $(\mathrm{A}, \mathrm{lb})$ erhält man durch Vergleich der sich so ergebenden Gleichungen mit den Kontinuitätsgleichungen (25 a) und ( $25 \mathrm{~b}$ ) die Ionisations- und Rekombinationsraten

$J_{1}=S_{1 \rightarrow \mathrm{K}}+S_{1 \rightarrow 2} \frac{S_{2 \rightarrow \mathrm{K}}}{S_{2 \rightarrow 1}+S_{2 \rightarrow \mathrm{K}}+g(1) A_{1,2 \rightarrow 1}}$,

(A, 3a)

und

$R_{2}=\alpha_{\mathrm{K} \rightarrow 1}+\alpha_{\mathrm{K} \rightarrow 2} \frac{S_{2 \rightarrow 1}+g(1) A_{1,2 \rightarrow 1}}{S_{2 \rightarrow 1}+S_{2} \rightarrow \mathrm{K}+g(1) A_{1,2 \rightarrow 1}}$.

$(\mathrm{A}, 3 \mathrm{~b})$
Dabei berücksichtigen die Stoßionisationsrate $S_{2 \rightarrow \mathrm{K}}$ und die Stoß-Strahlungsrekombinationsrate $\alpha_{K \rightarrow 2}$, daß die Ionisation vom ersten angeregten Zustand und die Rekombination in diesen Zustand auch über Stoß- und Strahlungsprozesse aus der Gruppe der höher angeregten Zustände als Zwischenstufe erfolgen. Die Raten für Ionisation vom Grundzustand und Rekombination in den Grundzustand sind durch $S_{1 \rightarrow \mathrm{K}}$ und $\alpha_{\mathrm{K} \rightarrow 1}$ und die Raten für Stoßanregung und -abregung des ersten angeregten Zustandes durch $S_{1 \rightarrow 2}$ und $S_{2 \rightarrow 1}$ gegeben.

Der in Gl. (A, 3a) und (A, 3b) auftretende Faktor $g$ (1) wird von MCWHIRTER ${ }^{19}$ in einer Tabelle (Tab. IV in $\left.{ }^{19}\right)$ in Abhängigkeit von der optischen Tiefe $\tau_{0}=\varkappa_{0} D$ im Zentrum der betrachteten Doppler-verbreiterten Linie angegeben. Dabei ist $\varkappa_{0}$ der Absorptionskoeffizient im Linienzentrum und $D$ eine für die Plasmaschicht charakteristische Länge. Es ergibt sich, daß im interessierenden Bereich des hier betrachteten Plasmas der in Frage kommende Wert von $g(1) A_{1,2 \rightarrow 1}$ bedeutend kleiner als die Rate $S_{2} \rightarrow \mathrm{K}$, jedoch größer als die Rate $S_{2 \rightarrow 1}$ ist. Ein Fehler bei der Bestimmung des Faktors $g(1)$ hat daher nur einen unwesentlichen Einfluß auf

19 R. W. P. McWhirTer, in: Plasma Diagnostic Techniques, Chapter 5, Academic Press, New York-London 1965, herausgeg. von R. H. Huddlestone u. S. L. LeONARd. 


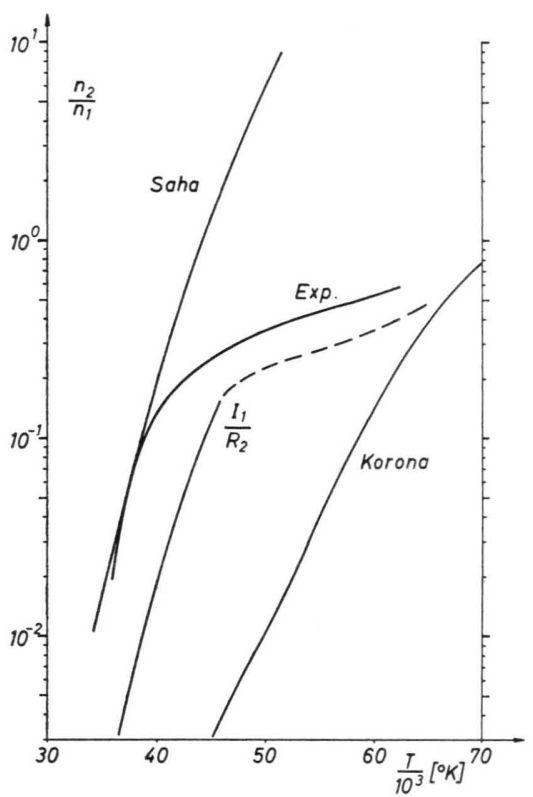

Abb. 5. Vergleich des gemessenen Ionisationsverhältnisses $\left(n_{2} / n_{1}\right)$ Exp von zweifach und einfach geladenen Heliumionen mit den aus statischen Ionisationsformeln - nämlich Gl. (26) mit den speziellen Raten $J_{1}$ und $R_{2}$ für dieses Plasma (Übergang zum optisch dünnen Fall gestrichelt), Saha-Gleichung und Koronaformel - berechneten Werten.

die Größe der Ionisationsrate $J_{1}$ [vgl. Gl. (A, 3a)], läßt aber den berechneten Wert der Rekombinationsrate $R_{2}$ [vgl. Gl. (A, 3b)] fehlerhaft werden. Man darf nach entsprechenden Abschätzungen erwarten, daß der auf diese Weise entstehende relative Fehler von $R_{2}$ im Radiusbereich $0,65 \leqq r \leqq 0,9 \mathrm{~cm}$ sicher $50 \%$ nicht überschreiten wird.

In Abb. 5 sind das gemessene Ionisationsverhältnis $\left(n_{2} / n_{1}\right)_{\text {Exp }}$ und das mit den jetzt bestimmten Raten $J_{1}$ und $R_{2}$ aus der statischen Beziehung (26) berechnete Ionisationsverhältnis $n_{2} / n_{1}=J_{1} / R_{2}$ als Funktion der Elektronentemperatur dargestellt, wobei der Übergang des statischen Verhältnisses zum optisch dünnen Fall gestrichelt gekennzeichnet ist. Außerdem sind die aus der Saha-Gleichung und der Koronaformel berechneten statischen Verläufe angegeben. Das Ionisationsverhältnis $\left(n_{2} / n_{1}\right)_{\text {Exp }}$ weicht im betrachteten nichtstatischen Plasma also im gesamten Meßbereich deutlich vom statischen Wert $J_{1} / R_{2}$ ab und ist daher durch die Kontinuitätsgleichungen (25 a) und ( $25 \mathrm{~b}$ ) bestimmt.

Diese Arbeit wurde im MPI für Physik in der Experimentellen Abteilung von Herrn Dr. v. Gierke begonnen und dann in Garching im Rahmen des Vertrages zwischen dem Institut für Plasmaphysik GmbH und der Europäischen Atomgemeinschaft über die Zusammenarbeit auf dem Gebiete der Plasmaphysik weitergeführt.

Herrn Professor Dr. R. WienECKE bin ich für fördernde Hinweise dankbar. Für zahlreiche anregende Diskussionen danke ich Herrn Dr. H. WulfF. 